\title{
A Novel Bi-enzyme Electrochemical Biosensor for Selective and Sensitive Determination of Methyl Salicylate
}

\author{
Yi Fang, Yogeswaran Umasankar and Ramaraja P. Ramasamy* \\ Nano Electrochemistry Laboratory, College of Engineering, University of Georgia, Athens, GA \\ 30602. \\ *Corresponding Author \\ Email: rama@uga.edu \\ Fax: 1-706-542-3804
}




\section{Abstract}

2 An amperometric sensor based on a bi-enzyme modified electrode was fabricated to detect

3 methyl salicylate, a volatile organic compound released by pathogen-infected plants via systemic

4 response. The detection is based on cascadic conversion reactions that result in an amperometric

5 electrochemical signal. The bi-enzyme electrode is made of alcohol oxidase and horseradish

6 peroxidase enzymes immobilized on to a carbon nanotube matrix through a molecular tethering

7 method. Methyl salicylate undergoes hydrolysis to form methanol, which is consumed by alcohol

8 oxidase to form formaldehyde while simultaneously reducing oxygen to hydrogen peroxide. The

9 hydrogen peroxide will be further reduced to water by horseradish peroxidase, which results in

10 an amperometric signal via direct electron transfer. The bi-enzyme biosensor was evaluated by

11 cyclic voltammetry and constant potential amperometry using hydrolyzed methyl salicylate as

12 the analyte. The sensitivity of the bi-enzyme biosensor as determined by cyclic voltammetry and

13 constant potential amperometry were 112.37 and $282.82 \mu \mathrm{A} \mathrm{cm}^{-2} \mathrm{mM}^{-1}$ respectively, and the

14 corresponding limits of detection were 22.95 and $0.98 \mu \mathrm{M}$ respectively. Constant potential

15 amperometry was also used to evaluate durability, repeatability and interference from other

16 compounds. Wintergreen oil was used for real sample study to establish the application of the bi-

17 enzyme sensor for selective determination of plant pathogen infections.

19 Keywords: agricultural biosensor, bi-enzyme electrode, methyl salicylate, alcohol oxidase, 20 horseradish peroxidase. 


\section{Introduction}

Agricultural economic losses due to pest and pathogen infections amount to $\$ 40$ billion

23 annually in the U.S. alone (Roberts et al. 2006). Minimizing crop damages through proper

24 disease management practices demand new technologies for detecting infections at very early

25 stages. Currently there is no rapid detection method available to detect the existence of plant

26 diseases on the field. Among the various off-field methods explored for the detection of

27 pathogenic diseases in crops, profiling of voltaic organic compounds (VOCs) released by plants

28 has been identified as an useful route to non-destructively analyze the sample. The chemical

29 signature of infected plants contains unique information related to the nature of the infection and

30 hence can be used as a reliable marker to identify pathogenic infections in crops. An infected

31 plant would produce different amount of VOCs as opposed to a healthy plant (Fang et al. 2014).

32 The VOCs in infected plants are produced through various biosynthetic pathways, including the

33 octadecanoid pathway leading to fatty-acid derived green leaf volatiles (GLVs), monoterpenes,

34 diterpenes, sesquiterpenes, isothiocyanates and a large diversity of aromatic metabolites

35 (Schoonhoven et al. 2005). Among the various compounds in the volatile signature of plants,

36 methyl salicylate (MeSA) is released in large quantities during pathogenic infections and

37 infestation and therefore is a suitable target compound (marker) for detecting biotic stresses of

38 plants. Plants produce MeSA through Shikimate biosynthesis pathway, during a biotic stress

39 event such as the pathogenic infection and herbivorous infestation. For instance, the production

40 of MeSA was observed from Tetranychus urticae infested lima beans. (De Boer and Dicke 2004;

41 De Boer et al. 2004; James and Price 2004; Pickett et al. 2006). MeSA production was also

42 reported from maize and pepper infected by Fusarium and Phytophthora capsici respectively

43 (Buttery et al. 1969; Piesik et al. 2011). MeSA is an allelochemical that is released not just at the 
44 site of pathogen infection but throughout the plant through a systematic response (Dudareva et al.

45 2006; Pichersky et al. 2006). It is one of the key markers for volatile based detection of fungal

46 diseases such as fruit blight, leaf blight, leather rot etc., which primarily affect the cucurbit crops.

47 Therefore it is a suitable target analyte (marker) for development of biosensors to detect

48 pathogenic diseases in crops. Moreover, the hydrolyzed form of MeSA (consisting methanol) can

49 be amperometrically detected on an enzymatic bioelectrode, providing the possibility of highly

50 selective quantitative detection based on bio-electrochemical redox reactions. Although gas

51 chromatography-mass spectrometry (GC-MS) is the most commonly used technique to study the

52 volatile signature of infected plants, this technique requires the sample to be collected in the field

53 and analyzed elsewhere in a laboratory (Ewen et al. 2004; Lui et al. 2005; Prithiviraj et al. 2004;

54 Vikram et al. 2006). Moreover the high cost of the instrumentation and complexity of the

55 analysis prohibit it from being used in the field for real time monitoring of plant diseases (Fang

56 and Ramasamy 2015). On the other hand amperometric electrochemical sensors such as the one

57 described in this work, offer many unique advantages for this application due to the following

58 characteristics: accuracy, rapid detection, robustness, non-invasive detection, selective and ultra-

59 low detection limits (Bakker 2004). They cost less and require no skilled analysts to infer the 60 results.

61 In this article, we report the successful development of a bi-enzyme based amperometic

62 biosensor for selective determination of methyl salicylate. The biosensor platform is a screen-

63 printed electrode, the surface of which is modified with multiwalled carbon nanotubes (CNTs).

64 The CNT acts as both immobilization support for the enzymes and as amperometric transducer.

65 The bio-recognition element consists of two enzymes namely alcohol oxidase (AOD) and

66 horseradish peroxidase (HRP) immobilized on a matrix of multiwalled CNTs. The 
67 immobilization was achieved through an established molecular tethering approach developed and

68 extensively demonstrated by us for other bio-electrochemical systems (Calkins et al. 2013; Lau

69 et al. 2012; Ramasamy et al. 2010; Umasankar et al. 2014; Umasankar and Ramasamy 2013). As

70 illustrated in Figure 1, the detection is carried out in three steps. First, MeSA is hydrolyzed in

71 potassium hydroxide $(\mathrm{KOH})$ to form potassium salicylate $(\mathrm{SA})$ and methanol $(\mathrm{MeOH})$. The $\mathrm{pH}$

72 was adjusted to 7.6 by adding phosphoric acid. Next the enzyme alcohol oxidase (AOD) converts

73 methanol into formaldehyde via its native biochemical reaction, during which a simultaneous

74 reduction of $\mathrm{O}_{2}$ to $\mathrm{H}_{2} \mathrm{O}_{2}$ takes place (Ozimek et al. 2005; Patel et al. 1981). The third step

75 involves the bio-electrochemical reduction of $\mathrm{H}_{2} \mathrm{O}_{2}$ to water using the second enzyme

76 horseradish peroxidase (HRP), which results in an amperometric signal for detection on the

77 electrode (Akkara et al. 1991; Ghindilis et al. 1997; Veitch 2004). The amperometric signal is

78 proportional to the concentration of the hydrolyzed MeSA in the electrolyte. The setup offers a

79 simple bi-enzyme biosensor for the detection of an important plant allelochemical namely methyl 80 salicylate.

\section{2. Materials and Methods}

\section{$83 \quad 2.1$ Materials}

Alcohol oxidase (EC 1.1.3.13) from Pichia pastoris was purchased from Sigma-Aldrich

85 and used as received. Horseradish peroxidase (specific activity $281 \mathrm{U} / \mathrm{mg}$ ) was purchased from

86 Calbiochem Inc. Multiwalled carbon nanotube (CNT) was obtained from DropSens Inc.

87 Pyrenebutanoic acid succinimidyl ester (PBSE) was purchased from AnaSpec Inc. (Fremont CA).

88 Dimethylformamide (DMF) was purchased from Acros Organics Inc. Chemicals for the 
89 interference study such as cis-3-hexenol, hexyl acetate and cis-hexenyl acetate were obtained

90 from TCI America (Portland, OR) and used as received. Wintergreen oil purchased from Piping

91 Rock Health Products was used as obtained for the real sample study. Methyl salicylate (MeSA)

92 was used as received from Sigma-Aldrich Inc. All other chemicals used in the work were of the

93 analytical grade. $100 \mathrm{mM}$ phosphate buffer (PB) ( $\mathrm{pH}$ 7.6) was used as the electrolyte for all

94 experiments. All the aqueous solutions were prepared using 18.2 $\mathrm{M} \Omega$ nano pure de-ionized (DI)

95 water. Electrolyte (buffer) solutions were oxygenated by purging with pure oxygen for $15 \mathrm{~min}$

96 before each experiment.

$97 \quad 2.2$ Apparatus

98 Cyclic voltammetry (CV) and constant potential amperometry were performed using CHI

99 920c potentiostat. A conventional three-electrode system consisting a Pt wire as the counter

100 electrode and $3 \mathrm{M} \mathrm{Ag} / \mathrm{AgCl}$ as the reference electrode was used for electrochemical

101 measurements. The working electrodes were glassy carbon (GC) electrode, modified multiwalled

102 CNT or a glassy carbon rotating disk electrode (RDE) from Pine Instrument Inc. All experiments

103 were carried out at $25 \pm 2{ }^{\circ} \mathrm{C}$.

$104 \quad 2.3$ Electrode preparation and electrochemical measurement

105 GC and RDE electrodes were surface cleaned by polishing on a polishing pad with 0.05

106 micron alumina polishing powder before each experiment. The electrodes were then cleaned

107 using ultrasonic cleaner and rinsed by DI water to remove the fine alumina powder adhered to

108 the electrode surface. The CNT suspension was prepared by ultrasonication of $1 \mathrm{mg}$ of

109 multiwalled CNTs in $1 \mathrm{~mL}$ DMF for 1 hour. The CNT modified electrodes were prepared by

110 drop casting $8 \mu \mathrm{L}$ (in 8 steps of $1 \mu \mathrm{L}$ ) for GC electrode and $12 \mu \mathrm{L}$ (in 3 steps of $4 \mu \mathrm{L}$ ) for RDE

111 followed by drying at $70{ }^{\circ} \mathrm{C}$. CNT modified electrodes were placed on the ice and allowed to 
112 cool down before $2+4 \mu \mathrm{L}$ of $10 \mathrm{mM}$ PBSE in DMF were added on GC and RDE respectively.

113 The electrodes were incubated for $15 \mathrm{~min}$ to allow the formation of non-covalent linkage

114 between CNT and PBSE. Then the electrodes were rinsed by DMF and $100 \mathrm{mM}$ PB (pH 7.6)

115 sequentially to remove excessive PBSE (Ramasamy et al. 2010). HRP solution was prepared by

116 dissolving $5 \mathrm{mg}$ HRP in $1 \mathrm{~mL} 20 \mathrm{mM} \mathrm{PB}$ (pH 7.6). The bi-enzyme solution was simply prepared

117 by mixing $5 \mu \mathrm{L}$ of alcohol oxidase solution and $5 \mu \mathrm{L}$ HRP solution. $10 \mu \mathrm{L}$ of bi-enzyme solution

118 was drop casted on the electrodes and incubated on ice for 30 min for enzyme immobilization.

119 The electrodes were rinsed with $100 \mathrm{mM} \mathrm{PB}(\mathrm{pH}$ 7.6) to remove any unimmobilized enzyme.

120 For CV measurements, the potential range used was from $0.2 \mathrm{~V}$ to $0.7 \mathrm{~V}$ at a scan rate of $20 \mathrm{mV}$

$121 \mathrm{~s}^{-1}$ with a sample interval of $0.001 \mathrm{~V}$. The initial potential for constant potential amperometry

122 with RDE was $0.45 \mathrm{~V}$ with $0.1 \mathrm{~s}$ interval for data collection.

\section{$123 \quad 2.4$ Hydrolysis of methyl salicylate}

124 The MeSA was mixed with $0.19 \mathrm{M} \mathrm{KOH}$ in $15 \mathrm{~mL}$ falcon tube to balance the ionic

125 strength of the buffer for hydrolysis. The falcon tube was sealed and placed in boiling water bath

126 for 30-minute hydrolysis. Then the falcon tube was cooled down to room temperature before

127 adding phosphoric acid to adjust the $\mathrm{pH}$ to 7.6 († supplementary data Fig. S1).

\section{3. Results and Discussion}

\section{$130 \quad 3.1$ Cyclic voltammetry on bi-enzyme modified electrode}

131 As methanol is the product of the hydrolysis step and the first reactant in the enzymatic

132 conversion reactions, the bi-enzyme system was evaluated first for methanol detection using 3

$133 \mathrm{mM}$ of methanol in the electrolyte. For comparison purposes, a CNT modified GC electrode 
134 functionalized with only one enzyme (HRP or AOD) as bio-recognition element was also

135 evaluated as two different controls. As shown by the cyclic voltammograms in Figure 2(a), the

136 amperometric signal indicated by the reduction wave originating at $0.6 \mathrm{~V}$ (with a peak at $0.45 \mathrm{~V}$ )

137 was generated only when both enzymes are present on the electrode. The cathodic wave

138 corresponds to the electrochemical reduction of hydrogen peroxide to water, which is the last

139 step of the cascade reactions as described in the Figure 1. No such reduction was observed in

140 Figure 2(a), when any one of the two enzymes was absent on the electrode. The results indicate

141 that the cascade reactions do not proceed if one of the enzymes was absent and that the hydrogen

142 peroxide is formed as a result of the cascade reactions. Moreover there was no peak attributable

143 to direct methanol oxidation at the electrode was observed in any of the voltammograms in

144 Figure 2(a), suggesting that the amperometric signal could be solely attributed to the hydrogen

145 peroxide reduction reaction.

146 The next step in the analysis is to evaluate if the intermediate compounds in the cascade

147 reactions namely potassium salt of salicylate (SA) and formaldehyde would contribute to the

148 amperometric signal in the region of interest $(0.6 \mathrm{~V}$ and below along the cathodic wave in Figure

149 2(a)). For this, the HRP and AOD immobilized electrodes were tested using CV in the same

150 potential window using reagent solutions of salicylate (SA) and formaldehyde solutions

151 respectively and the corresponding voltammograms are given in the supplementary data $\uparrow$ Figure

152 S2. The results in Figure S2(a) suggest that formaldehyde did not contribute to the reduction

153 current in the potential range of interest. The SA however, exhibits a small redox peak around

$1540.5 \mathrm{~V}$, but the signal is weak and much smaller compared to the electrochemical signal for

155 hydrogen peroxide reduction († supplementary data Fig. S2) (Zhang et al. 2010). There was also

156 a large oxidation peak for salicylate oxidation above $0.65 \mathrm{~V}$ noticed in the voltammograms in 
157 Figure S2. However this oxidation current does not interfere with the reduction of hydrogen

158 peroxide, which happens only below $0.6 \mathrm{~V}$. It can also be observed that SA does not interfere the

159 current signal collected from methanol on bi-enzyme electrode ( $\dagger$ supplementary data Fig. S2(b)).

160 The results shown in Figure 1(a) and Figure S2 demonstrate that the methanol (from hydrolyzed

$161 \mathrm{MeSA})$ is the only compound responsible for the amperometric signal observed in the

162 voltammograms. This serves as a positive control for our actual metahyl salicylate detection

163 described further below.

164 The next step in the analysis is to determine if MeSA can be detected amperometrically

165 through the cascade reactions with no initial methanol present in the system. For this purpose,

$1661.88 \mathrm{mM}$ hydrolyzed MeSA was used as the analyte in the electrochemical cell and CV was

167 performed in the same potential window. As shown in Figure 2(b), the voltammograms showed a

168 similar electrochemical response for the bi-enzyme electrode for the hydrolyzed MeSA analyte

169 as it was for methanol, with the hydrogen peroxide reduction peak occurring at $0.45 \mathrm{~V}$. The

170 electrodes modified with only one of the two enzymes as well as the electrode with no enzyme

171 modification exhibited no redox response suggesting that the amperometric signal is generated

172 only when both enzymes are present, to carry out all reactions in the cascade. The results also

173 confirm that the electrochemical reduction of hydrogen peroxide requires HRP, as there was no

174 distinct signal observed in the voltammograms for direct reduction of $\mathrm{H}_{2} \mathrm{O}_{2}$ in the absence of

175 HRP. Similarly no electrochemical signal was generated for direct oxidation of either methanol

176 or SA in the absence of enzymes. The results in Figure 2(b) demonstrate that only the bi-enzyme

177 modified electrode can detect the MeSA in the analyte. The optimal loading of enzymes on the

178 electrode was optimized by a series trial experiments with different mass ratios of both enzymes

179 (2.8 U AOD / 14.0 U HRP, 5.5 U AOD / 7.0 U HRP and 11.0 U AOD / 3.5 U HRP), the results 
180 of which are given in supplementary data $\uparrow$ Figure S3. The optimal mass ratio was determined to

181 be $5.5 \mathrm{U}$ of AOD and 7.0 $\mathrm{U}$ of HRP (1:1 mass ratio of AOD: HRP) on the CNT modified

182 electrode.

\section{$183 \quad 3.2$ Electrochemical response of the bi-enzyme sensor}

184 The amperometric response of the bi-enzyme modified electrode at different

185 concentrations of the analyte was analyzed using both CV and constant potential amperometry.

186 The 12 different concentrations of the hydrolyzed MeSA ranged from $1 \mu \mathrm{M}$ to $3 \mathrm{mM}$ and were

187 studied by stepwise addition. The system was equilibrated for 10 seconds after the stepwise

188 analyte addition at each concentration before a measurement was made. The above concentration

189 range was chosen based on a series of experiments, where the lowest limit was determined based

190 on the noticeable increase in reduction current upon an incremental addition of hydrolyzed

191 MeSA into the electrolyte. Similarly, the upper concentration limit was chosen based on the rate

192 of decrease in oxidation current during subsequent additions of hydrolyzed MeSA.

193 Voltammograms shown in Figure 3(a) indicate that before the addition of hydrolyzed MeSA no

194 redox peak was observed. When the concentration of hydrolyzed MeSA in the electrolyte was

195 increased by stepwise additions to $10 \mu \mathrm{M}$ and beyond, noticeable $\mathrm{H}_{2} \mathrm{O}_{2}$ reduction wave was

196 observed below $0.6 \mathrm{~V}$ with a peak around $0.45 \mathrm{~V}$. The peak current increased with increasing

197 concentrations of hydrolyzed MeSA in the electrolyte. When the concentration of hydrolyzed

198 MeSA exceeds $1 \mathrm{mM}$ an oxidation peak was also observed at $0.5 \mathrm{~V}$, which could be attributed to

199 the oxidation of SA present in the hydrolyzed MeSA. Amperometric sensor parameters such as

200 sensitivity, limit of detection (LOD), and limit of quantification (LOQ) can be obtained by the

201 following equations. 


$$
\begin{gathered}
\text { Sensitivity }=\frac{\text { Slope of calibritaion curve }\left(A M^{-1}\right)}{\text { Area of electrode }\left(\mathrm{cm}^{2}\right)} \\
L O D=3.3 \times \frac{S D \text { of peak current in the absence of analyte }(A)}{\text { Slope of linear calibration curve }\left(A M^{-1}\right)} \\
L O Q=10 \times \frac{\text { SD of peak current in the absence of analyte }(A)}{\text { Slope of linear calibration curve }\left(A M^{-1}\right)}
\end{gathered}
$$

202 The data and values for the above parameters as well as the linear range of reliable detection and

203 initial threshold concentration for amperometric response were tabulated in the supplementary 204 data† Table S1.

205 Since CV response includes non-faradaic contributions, it does not provide a steady state 206 response of the amperometric sensor. Therefore in order to establish the steady state response of 207 the biosensor, constant potential amperometry (chronoamperometry) was also used to determine 208 the amperometric response. For this a RDE electrode modified with the bi-enzyme/CNT matrix 209 was used as the working electrode and the measurements were made at a rotational speed of $2101200 \mathrm{rpm}$ to eliminate mass transfer limitations. The bi-enzyme modified RDE was stabilized for 211300 seconds before the addition of hydrolyzed MeSA from $0.1 \mu \mathrm{M}$ to $1 \mathrm{mM}$ in stepwise 212 additions after each 50 seconds. The first observable stepwise increase appears at the 213 concentration of $0.5 \mu \mathrm{M}$ as it shown in Figure 3(b). The relevant electrochemical parameters 214 obtained from the amperometry data is also reported in supplementary data $\uparrow$ Table S1. The 215 results suggest a higher sensitivity for constant potential amperometry $\left(282.82 \mu \mathrm{A} \mathrm{cm}{ }^{-2} \mathrm{mM}^{-1}\right)$ as 216 opposed to that for cyclic voltammetry $\left(112.37 \mu \mathrm{A} \mathrm{cm} \mathrm{cm}^{-1}\right)$. Furthermore, the limit of 217 detection for constant potential amperometry can be lowered down to $0.98 \mu \mathrm{M}$ as opposed to 218 cyclic voltammetry $(22.95 \mu \mathrm{M})$, which is reported in supplementary data $\uparrow$ Figure S4. To put this

219 LOD into perspective, a typical MeSA release rate by a plant is $283 \mathrm{ng} \mathrm{hr}^{-1}$ plant $^{-1}$ (Shulaev et al. 
220 1997). At this typical rate, one could accumulate enough sample from the plant for reliable

221 detection within 1.05 hours during which about $0.98 \mu \mathrm{M}$ of MeSA can be accumulated in a $2 \mathrm{~mL}$

222 electrochemical cell (supplementary data† Table S1). Though the linear range for constant

223 potential amperometry is narrower than $\mathrm{CV}$, it is a preferable amperometric technique over $\mathrm{CV}$

224 based on other estimated parameters.

\section{$225 \quad 3.3$ Interference studies}

MeSA is not the only VOC produced by plants during biotic stress events. Other VOCs

227 such as green leaf volatiles (GLVs) that are non-specific to the pathogen infections are also often

228 released at high concentrations, which could cause interference during MeSA measurement.

229 Therefore a representative set of such GLV compounds namely, cis-3-hexenol, hexyl acetate and

230 cis-3-hexenyl acetate were used to determine the effect of interference on the biosensor response

231 using constant potential amperometry. The bi-enzyme modified RDE was preconditioned until

232300 seconds and hydrolyzed MeSA was added to maintain $50 \mu \mathrm{M}$ of working concentration,

233 which is in the middle of linear range. The RDE was further stabilized for 500 seconds before

234 adding different concentrations of hydrolyzed GLVs from $10 \mu \mathrm{M}$ to $1 \mathrm{mM}$ (final concentration in

235 the electrolyte) at 50-second intervals. The experiment was conducted for all three GLVs as well

236 as the same volume of $100 \mathrm{mM}$ phosphate buffer $(\mathrm{pH}$ 7.6) as the control. The raw interference

237 data of current vs. time is reported in supplementary data $\uparrow$ Figure S5. While the typical GLV

238 concentration is about $10 \mu \mathrm{M}$ for cis-3-hexenol, $20 \mu \mathrm{M}$ for cis-hexenyl acetate and $1 \mu \mathrm{M}$ for

239 hexyl acetate (Umasankar et al. 2012), a wider concentration range (0 to $1 \mathrm{mM}$ ) of these

240 interfering compounds were tested to simulate extreme scenarios (Danner et al. 2011).

Figure 4(a) displays the results from the interference studies, where the variation in the

242 amperometric signal of the sensor was plotted against time during stepwise additions of each 
243 interfering compound, namely cis-3-hexenol, hexyl acetate and cis-3-hexenyl acetate. To account

244 for the effects that arise due to the volume change of the electrolyte, a control interference study

245 was performed, where phosphate buffer (PB) was added stepwise to mimic the volume change in

246 the electrolytes. The decrease in the current values during stepwise addition of PB in control

247 experiment could be attributed to the dilution effects arising from the stepwise addition, as the

248 buffer itself does not contribute to interference. On the other hand, amperometric signal in the

249 presence of GLV were slightly higher compared to the control. Figure 4(b) shows the percentage

250 of interference at different concentrations of the interfering compounds, which ranges from $0 \%$

251 (for $0 \mu \mathrm{M}$ of GLVs) to $2.36 \%, 2.14 \%$ and $3.43 \%$ respectively, (for $100 \mu \mathrm{M}$ of cis-3-hexenol,

252 hexyl acetate and cis-3-hexenyl acetate) as reported in supplementary data† Table S2. Since 100

$253 \mu \mathrm{M}$ of GLVs represent is about two orders of magnitude higher than typical concentrations of

254 GLVs, we could conclude that bi-enzyme sensor for MeSA detection suffers minimally from the

255 interference of GLVs at their typical concentrations in plant volatile signature.

256 In addition to the above-mentioned compounds, methanol, which produced by most of C3

257 plants, could also potentially interfere with MeSA signal, since the detection of MeSA is based

258 on methanol that was formed after hydrolysis (Fall and Benson 1996). Therefore, the sensor is

259 primarily meant for use with $\mathrm{C} 4$ plants such as corn. Previous research has indicated that

260 significant amount of MeSA was released by juvenile corn (Zea mays) while methanol

261 production was not reported (Bernasconi et al. 1998; MacDonald and Fall 1993). The existing

262 biosensor could be applied for crops like tobacco, as the quantity of methanol released by the

263 tobacco crop is negligible compared to the quantity of MeSA (Bernasconi et al. 1998; Shulaev et

264 al. 1997). Moreover pre-treatment of the volatile sample to remove methanol vapors could also 
265 be applied to avoid false positive signals from methanol vapors. (Premkumar and Krishnamohan 266 2010).

\section{3.4 Durability and repeatability}

268 Durability of the bi-enzyme biosensor was evaluated for 10 days and the original data is 269 reported ( $\dagger$ supplementary data Table S3). The duration of the durability study was decided 270 based on the duration it takes to notice a significant decrease in the amperometric signal. For this, 271 constant potential amperometry was used to detect $50 \mu \mathrm{M}$ hydrolyzed MeSA using the bi-

272 enzyme modified RDE, where the net current density was measured before and after addition of 273 the hydrolyzed MeSA. The RDE was then stored in $100 \mathrm{mM} \mathrm{PB}(\mathrm{pH} 7.6)$ at $4{ }^{\circ} \mathrm{C}$. The 274 experiments were performed on day $0,1,2,4,7$ and 10 by using the same procedure. The result 275 of the durability shows that the current density for $\mathrm{H}_{2} \mathrm{O}_{2}$ reduction decreased gradually over the 7 276 days (supplementary data† Fig. S6). The day when initial measurements were made was 277 considered as day 0 . On day 1 , the current dropped from $100 \%$ to $95 \%$. However, between day 2781 and 4 , the electrode behaved relatively stable with very little variations. The amperometric 279 signal current was above $90 \%$ of the initial value even after 7 days. However, on day 10 , the 280 current sharply decreased to $79 \%$, which could be attributed to the loss of enzyme activity 281 (supplementary data $\uparrow$ Table S3). Since the sensor electrodes are meant for single-use testing, the 282 durability data here shows satisfactory retention of signal durability by the bi-enzyme sensor for 2837 days.

Tests were also conducted to evaluate the repeatability of the bi-enzyme biosensor

285 performance. The experiments were conducted on the bi-enzyme modified RDE using identical 286 concentrations of $50 \mu \mathrm{M}$ hydrolyzed MeSA (supplementary data† Fig. S7). The current density 287 differed slightly between each measurement, which could be attributed to the slight change in 
enzyme orientation on the surface of the electrode. Upon the immobilization, the enzyme could

289 orient itself on the surface in multiple ways, and therefore the active site of the enzyme could be

290 either exposed to the electrolyte side or facing the electrode. The variation in the orientation

291 could impact substrate binding and reaction thereby causing small variations in the

292 electrochemical parameters measured in each experiment. Difference in the currents before and

293 after adding hydrolyzed MeSA was used for relative standard deviation (RSD) calculation. The

294 RSD for the repeatability experiments was $6.6 \%$ († supplementary data Table S4), which is

295 quite acceptable based on for enzyme-based sensors.

\section{$296 \quad 3.5$ Real Sample Study}

Real sample study was conducted in order to evaluate the bi-enzyme biosensor on a

298 native analyte that contain methyl salicylate. Wintergreen oil essentially consists $98 \%$ of MeSA,

299 which is produced by the enzymes of the wintergreen when it is under stress. Wintergreen oil

300 was hydrolyzed as stated before and used as the analyte for real-sample studies. However, MeSA,

301 which is the main compound in wintergreen oil, is not present in the plant initially. It is only

302 produced enzymatically from a glucoside within the leaves when they are macerated in warm

303 water. In this experiment, wintergreen oil was introduced to simulate the situation when plant

304 produces MeSA due to plant infection. Different quantities of hydrolyzed wintergreen oil

305 containing 98\% MeSA was added stepwise to result in concentrations of $0.1,0.5,1,2.5,5,10,25$,

30650 and $100 \mu \mathrm{M}$ were added into electrochemical cell and the amperometric signal was measured

307 and compared against the amperometric signal of pure MeSA standards, in order to determine

308 the estimated concentration of MeSA in the wintergreen oil. Recovery was calculated from the

309 estimated concentration obtained from the working curve of standard MeSA sample and the

310 known standard concentration. 
As shown in Figure 5, three replicates of measurements were obtained using hydrolyzed

312 wintergreen oil and the trend was found to be the same as that of pure MeSA. As seen in Table 1,

313 the RSD values ranged from $91 \%$ to $122 \%$. The bias could arise due to the presence of other

314 electrochemical active compounds in wintergreen oil such as $\alpha$-pinene, myrcene, $\delta$ - 3 -carene,

315 limonene, 3,7-guaiadiene and $\delta$-cadinene reacted directly on electrode (Gurung 2007). From the

316 RSD values in Table 1, it can be concluded that the real sample experiments were highly

317 repeatable when the concentration is close to the middle point of the linear range (e.g. $49 \mu \mathrm{M})$.

318 On the other hand, the repeatability decreased when concentrations were at the lower limit (2.45

$319 \mu \mathrm{M})$ and upper limit $(98 \mu \mathrm{M})$ of the linear range.

321 4. Conclusion

A bi-enzyme based amperometric electrochemical biosensor has been developed using an

323 established immobilization method. The biosensor was successfully tested for the detection of

324 MeSA, a common VOC released by plants during pathogen infections. The sensor was

325 characterized using $\mathrm{CV}$ and constant potential amperometry. Constant potential amperometry

326 demonstrates higher sensitivity and lower limit of detection, which allows for faster sample

327 collection for MeSA detection. The interference study with three GLVs indicates that the

328 biosensor signal is not significantly affected by GLVs. The biosensor also displays satisfactory

329 durability and acceptable repeatability. Wintergreen oil was used for real sample study, for

330 demonstrating the on-field applicability of the sensor. Future work would focus on optimizing

331 the sensor performance in terms of sensitivity, durability and repeatability by adopting more

332 specific enzyme like salicylate hydroxylase and other enzyme stabilization methods. The

333 fabricated biosensor will be used to test the head-space gas produced by the plants in class bell 
334 jar in the prototype demonstrations and would eventually be used in the greenhouse. The work 335 demonstrates an application of an electrochemical sensor with bio-recognition element for 336 selective determination of plant pathogen infections based on methyl salicylate production. 


\section{Abbreviations Used}

338 AOD, alcohol oxidase; CNT, carbon nanotube; CV, cyclic voltammetry; DMF,

339 dimethylformamide; GC-MS, gas chromatography-mass spectrometry; GC, glassy-carbon; GLV, 340 green leaf volatile; HRP, horseradish peroxidase; LOD, limit of detection; LOQ, limit of 341 quantification; $\mathrm{MeOH}$, methanol; MeSA, methyl salicylate; PB, phosphate buffer; PBSE, 342 pyrenebutanoic acid succinimidyl ester; RDE, rotating disc electrode; RSD, relative standard 343 deviation; SA, salicylate; VOC, volatile organic compound.

\section{Acknowledgement}

346 We acknowledge the National Science Foundation (CBET-1159540), ACS Herman Frasch

347 Foundation (Grant \# 045097-01) and National Institute of Food and Agriculture (Grant \# 2015348 67021-23188) for financial support.

\section{Appendix A. Supplementary Information}

351 Additional tables and figures as cited in the text. Supplementary data associated with this article 352 can be found at the website.

\section{Author Information}

$355 *$ Corresponding Author: rama@uga.edu 


\section{References}

358 Akkara, J.A., Senecal, K.J., Kaplan, D.L., 1991. Synthesis and characterization of polymers produced by horseradish peroxidase in dioxane. Journal of Polymer Science Part A: Polymer 360 Chemistry 29(11), 1561-1574.

361 Bakker, E., 2004. Electrochemical sensors. Analytical chemistry 76(12), 3285-3298.

362 Bernasconi, M.L., Turlings, T.C., Ambrosetti, L., Bassetti, P., Dorn, S., 1998. Herbivore-induced 363 emissions of maize volatiles repel the corn leaf aphid, Rhopalosiphum maidis. Entomologia 364 Experimentalis et Applicata 87(2), 133-142.

365 Buttery, R., Seifert, R., Guadagni, D., Ling, L., 1969. Characterization of some volatile 366 constituents of bell peppers. J. Agric. Food Chem. 17(6), 1322-1327.

367 Calkins, J.O., Umasankar, Y., O'Neill, H., Ramasamy, R.P., 2013. High photo-electrochemical 368 activity of thylakoid-carbon nanotube composites for photosynthetic energy conversion. Energy \& Environmental Science 6(6), 1891-1900.

371

372 Danner, H., Boeckler, G.A., Irmisch, S., Yuan, J.S., Chen, F., Gershenzon, J., Unsicker, S.B., Köllner, T.G., 2011. Four terpene synthases produce major compounds of the gypsy moth feeding-induced volatile blend of Populus trichocarpa. Phytochemistry 72(9), 897-908.

373 De Boer, J.G., Dicke, M., 2004. The role of methyl salicylate in prey searching behavior of the 374 predatory mite Phytoseiulus persimilis. Journal of chemical ecology 30(2), 255-271.

375 De Boer, J.G., Posthumus, M.A., Dicke, M., 2004. Identification of volatiles that are used in 376 discrimination between plants infested with prey or nonprey herbivores by a predatory mite.

377 Journal of chemical ecology 30(11), 2215-2230.

378 Dudareva, N., Negre, F., Nagegowda, D.A., Orlova, I., 2006. Plant volatiles: recent advances and future perspectives. Critical reviews in plant sciences 25(5), 417-440.

380 Ewen, R.J., Jones, P.R., Ratcliffe, N.M., Spencer-Phillips, P.T., 2004. Identification by gas 381 chromatography-mass spectrometry of the volatile organic compounds emitted from the woodrotting fungi Serpula lacrymans and Coniophora puteana, and from Pinus sylvestris timber. Mycological research 108(A Pediatria07), 806-814.

384 Fall, R., Benson, A.A., 1996. Leaf methanol - the simplest natural product from plants. Trends in 385 Plant Science 1(9), 296-301.

386 Fang, Y., Ramasamy, R.P., 2015. Current and Prospective Methods for Plant Disease Detection. 387 Biosensors 5(3), 537-561.

388 Fang, Y., Umasankar, Y., Ramasamy, R.P., 2014. Electrochemical detection of p-ethylguaiacol, a fungi infected fruit volatile using metal oxide nanoparticles. Analyst 139(15), 3804-3810.

Ghindilis, A.L., Atanasov, P., Wilkins, E., 1997. Enzyme-catalyzed direct electron transfer: Fundamentals and analytical applications. Electroanalysis 9(9), 661-674.

393 Society, Dolakha, Nepal.

394 James, D.G., Price, T.S., 2004. Field-testing of methyl salicylate for recruitment and retention of 395 beneficial insects in grapes and hops. Journal of chemical ecology 30(8), 1613-1628. 
Lau, C., Adkins, E.R., Ramasamy, R.P., Luckarift, H.R., Johnson, G.R., Atanassov, P., 2012. Design of Carbon Nanotube-Based Gas-Diffusion Cathode for O2 Reduction by Multicopper Oxidases. Advanced Energy Materials 2(1), 162-168.

Lui, L., Vikram, A., Hamzehzarghani, H., Kushalappa, A., 2005. Discrimination of three fungal diseases of potato tubers based on volatile metabolic profiles developed using GC/MS. Potato Research 48(1-2), 85-96.

402 MacDonald, R.C., Fall, R., 1993. Detection of substantial emissions of methanol from plants to the atmosphere. Atmospheric Environment. Part A. General Topics 27(11), 1709-1713.

Ozimek, P., Veenhuis, M., Klei, I.J., 2005. Alcohol oxidase: a complex peroxisomal, oligomeric flavoprotein. FEMS yeast research 5(11), 975-983.

Patel, R.N., Hou, C., Laskin, A., Derelanko, P., 1981. Microbial oxidation of methanol: properties of crystallized alcohol oxidase from a yeast, Pichia sp. Archives of biochemistry and biophysics 210(2), 481-488.

Pichersky, E., Noel, J.P., Dudareva, N., 2006. Biosynthesis of plant volatiles: nature's diversity and ingenuity. Science 311(5762), 808-811.

411 Pickett, J.A., Bruce, T.J., Chamberlain, K., Hassanali, A., Khan, Z.R., Matthes, M.C., Napier, J.A., Smart, L.E., Wadhams, L.J., Woodcock, C.M., 2006. Plant volatiles yielding new ways to exploit plant defence. Chemical Ecology: from Gene to Ecosystem. Edited by Dicke M, Takken 414 W. Springer, 161-173.

415 Piesik, D., Lemńczyk, G., Skoczek, A., Lamparski, R., Bocianowski, J., Kotwica, K., Delaney, K.J., 2011. Fusarium infection in maize: Volatile induction of infected and neighboring uninfected plants has the potential to attract a pest cereal leaf beetle, Oulema melanopus. J. Plant Physiol. 168(13), 1534-1542.

Premkumar, R., Krishnamohan, N., 2010. Removal of methanol from waste gas using biofiltration. Journal of Applied Sciences Research(November), 1898-1907.

Prithiviraj, B., Vikram, A., Kushalappa, A., Yaylayan, V., 2004. Volatile metabolite profiling for the discrimination of onion bulbs infected by Erwinia carotovora ssp. carotovora, Fusarium oxysporum and Botrytis allii. European Journal of Plant Pathology 110(4), 371-377. electrocatalytic activity of tethered multicopper oxidase-carbon nanotube conjugates. Chemical Communications 46(33), 6045-6047.

Roberts, M.J., Schimmelpfennig, D.E., Ashley, E., Livingston, M.J., Ash, M., Vasavada, U., 2006. The value of plant disease early-warning systems: a case study of USDA's soybean rust coordinated framework. United States Department of Agriculture, Economic Research Service.

432 Shulaev, V., Silverman, P., Raskin, I., 1997. Airborne signalling by methyl salicylate in plant 433

Schoonhoven, L.M., Van Loon, J.J., Dicke, M., 2005. Insect-plant biology. Oxford University Press. 
434 Umasankar, Y., Brooks, D.B., Brown, B., Zhou, Z., Ramasamy, R.P., 2014. Three Dimensional 435 Carbon Nanosheets as a Novel Catalyst Support for Enzymatic Bioelectrodes. Advanced Energy 436 Materials 4(6).

437 Umasankar, Y., Rains, G.C., Ramasamy, R.P., 2012. Electroanalytical studies on green leaf 438 volatiles for potential sensor development. Analyst 137(13), 3138-3145.

439 Umasankar, Y., Ramasamy, R.P., 2013. On the bio-electrocatalytic activity of tyrosinase for 440 oxygen reduction reaction. Catal. Sci. Technol. 3(10), 2546-2549.

441 Veitch, N.C., 2004. Horseradish peroxidase: a modern view of a classic enzyme. Phytochemistry 442 65(3), 249-259.

443 Vikram, A., Lui, L., Hossain, A., Kushalappa, A., 2006. Metabolic fingerprinting to discriminate 444 diseases of stored carrots. Annals of applied biology 148(1), 17-26.

445 Zhang, W.-D., Xu, B., Hong, Y.-X., Yu, Y.-X., Ye, J.-S., Zhang, J.-Q., 2010. Electrochemical 446 oxidation of salicylic acid at well-aligned multiwalled carbon nanotube electrode and its 447 detection. Journal of Solid State Electrochemistry 14(9), 1713-1718. 


\section{Figure Captions}

Fig. 1: Schematic illustration of methyl salicylate detection by bi-enzyme modified electrode. The process begins with the hydrolysis of methyl salicylate to form salicylate and methanol (1), oxidation of methanol and production of hydrogen peroxide (2) and direct electron transfer from electrode to hydrogen peroxide by horseradish peroxidase (3).

Fig. 2: CV responses of $3 \mathrm{mM}$ methanol on bi-enzyme modified, AOD modified and HRP modified electrodes (a) and $1.88 \mathrm{mM}$ hydrolyzed methyl salicylate on bi-enzyme modified, AOD modified, HRP modified and no enzyme modified electrodes (b).

Fig. 3: $\mathrm{CV}$ responses of hydrolyzed methyl salicylate from $0,1 \mu \mathrm{M}$ to $3 \mathrm{mM}$ on bi-enzyme modified GC electrode (a). Insert in (a) shows current density versus concentration. Amperometry of hydrolyzed methyl salicylate from $0,0.1$ to $1 \mathrm{mM}$ on bi-enzyme modified RDE (b). Insert in (b) shows current density versus concentration.

Fig. 4: Interference of cis-3-hexenol, hexyl acetate and cis-3-hexenyl acetate on the amperometric measurements. Phosphate buffer is used as the control interfering compound (a). Percentage of interference caused by each compound at different concentrations (b).

Fig. 5: Constant potential amperometry of the electrode upon the stepwise additions of wintergreen oil (3 replicates) up to $100 \mu \mathrm{M}$ of (98\% MeSA) wintergreen oil. A control experiment using standard methyl salicylate (black curve) is also shown for comparison. 


\section{Tables}

Table 1: Standard concentration, calculated concentration, recovery, SD and RSD of hydrolyzed wintergreen oil for real sample study

\begin{tabular}{ccccc}
\hline Std. conc. $(\mu \mathrm{M})$ & Cal. conc. $(\mu \mathrm{M})$ & Recovery $(\%)$ & SD $(\mu \mathrm{M})$ & RSD $(\%)$ \\
2.45 & 2.24 & 91 & 0.28 & 12.38 \\
4.90 & 5.78 & 118 & 0.28 & 4.90 \\
9.80 & 12.64 & 129 & 0.29 & 2.29 \\
24.5 & 31.38 & 128 & 0.96 & 3.06 \\
49.0 & 59.81 & 122 & 2.71 & 4.53 \\
\hline
\end{tabular}




\section{Figures}

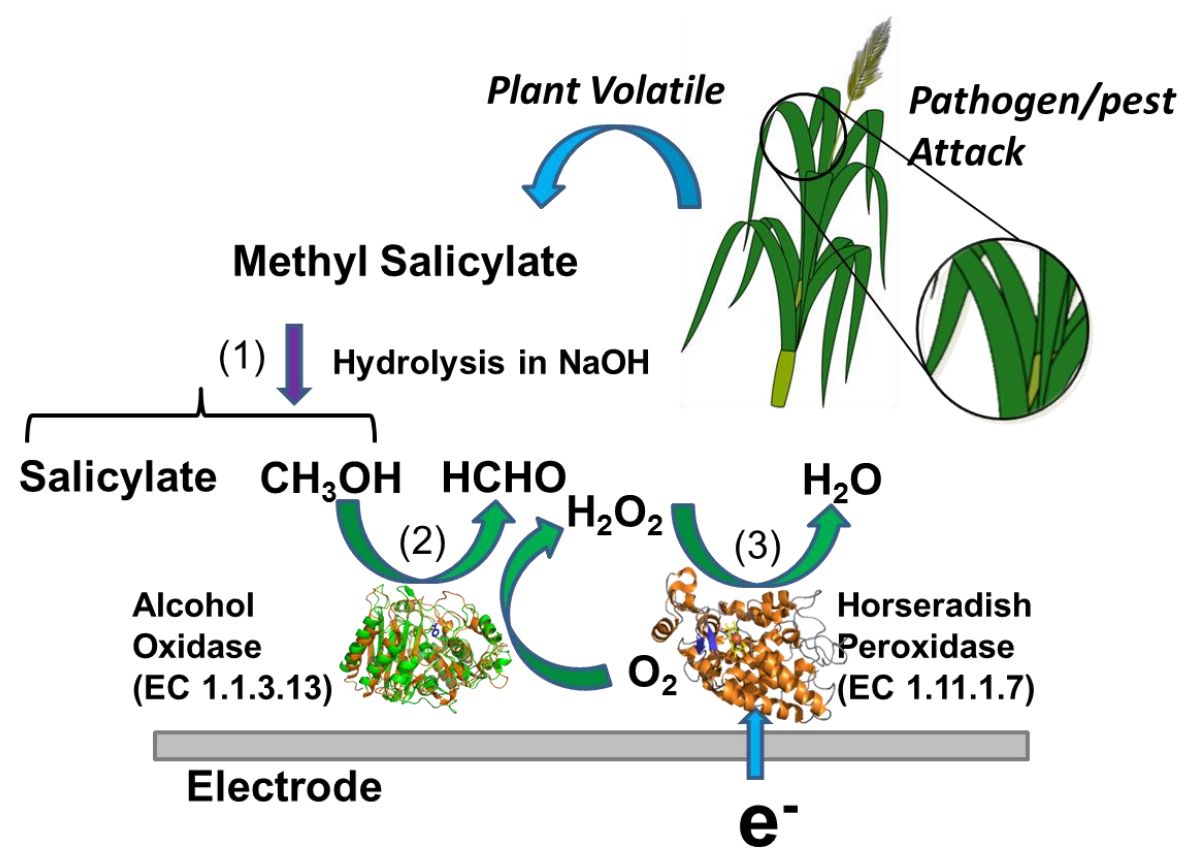

Figure 1: Schematic illustration of methyl salicylate detection by bi-enzyme modified electrode. The process begins with the hydrolysis of methyl salicylate to form salicylate and methanol (1), oxidation of methanol and production of hydrogen peroxide (2) and direct electron transfer from electrode to hydrogen peroxide by horseradish peroxidase (3). Not drawn to scale. 

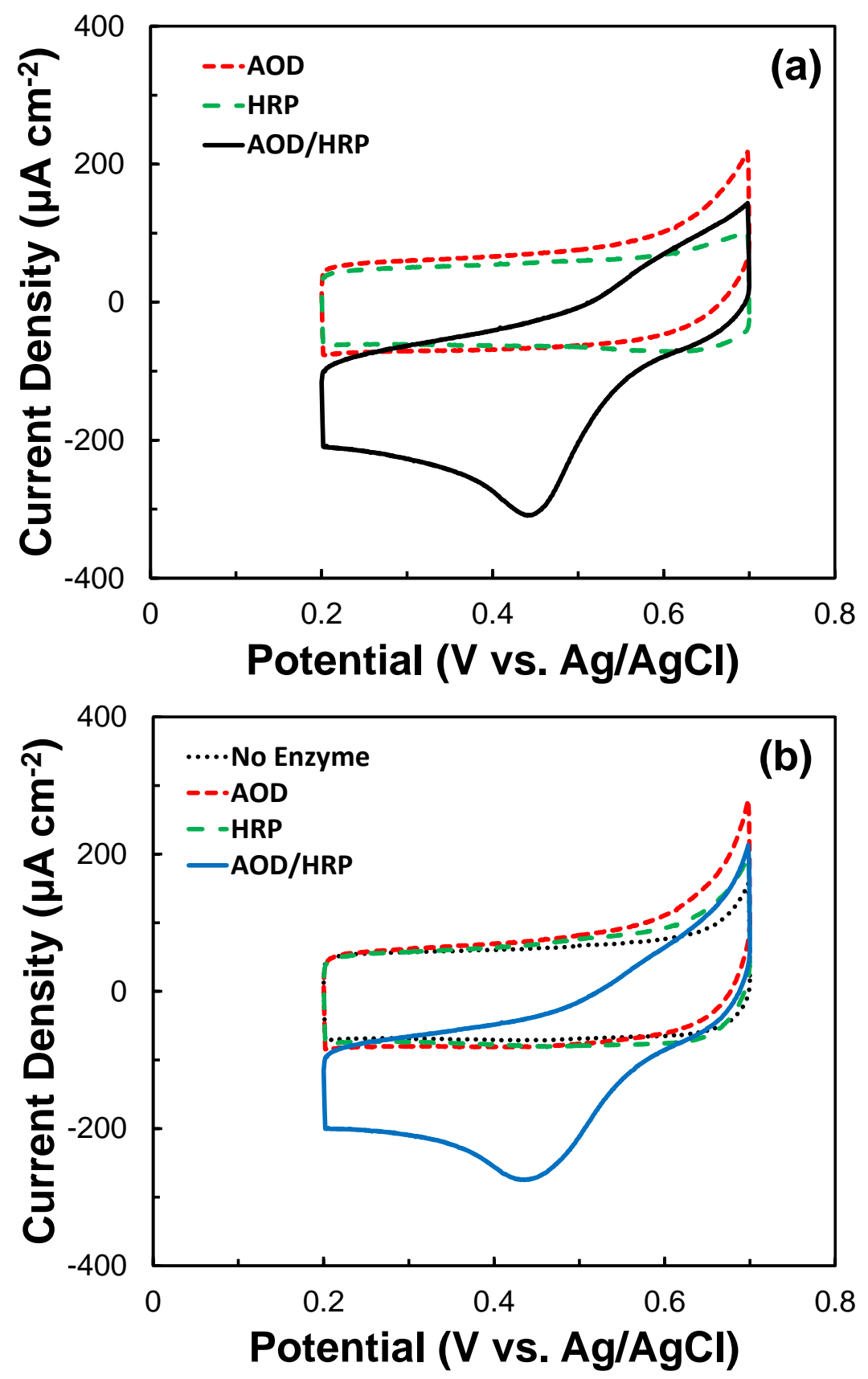

Figure 2: $\mathrm{CV}$ responses of $3 \mathrm{mM}$ methanol on bi-enzyme modified, AOD modified and HRP modified electrodes (a) and $1.88 \mathrm{mM}$ hydrolyzed methyl salicylate on bi-enzyme modified, AOD modified, HRP modified and no enzyme modified electrodes (b). 

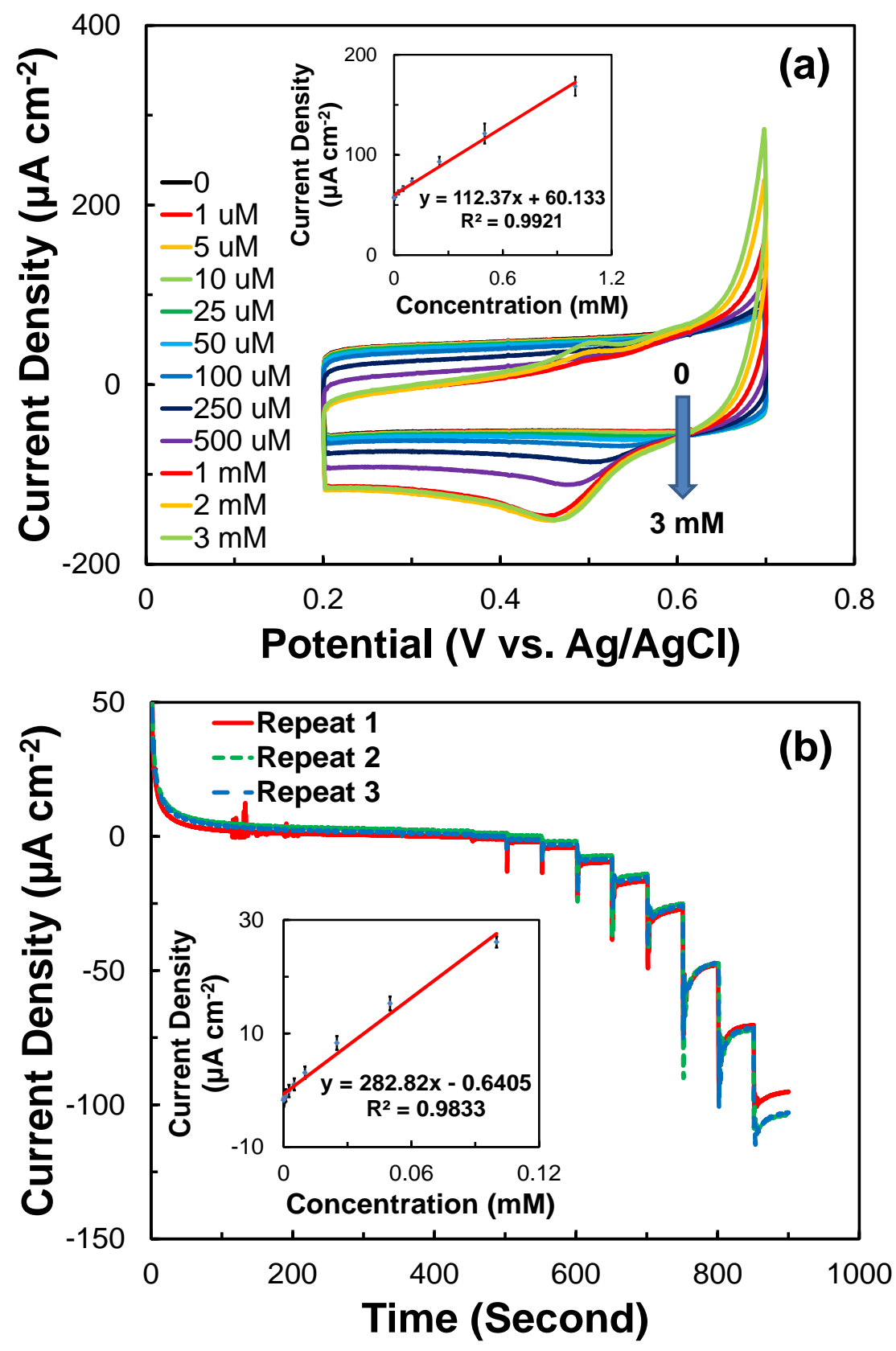

Figure 3: $\mathrm{CV}$ responses of hydrolyzed methyl salicylate from $0,1 \mu \mathrm{M}$ to $3 \mathrm{mM}$ on bi-enzyme modified GC electrode (a). Insert in (a) shows current density versus concentration. Amperometry of hydrolyzed methyl salicylate from $0,0.1$ to $1 \mathrm{mM}$ on bi-enzyme modified RDE (b). Insert in (b) shows current density versus concentration. 

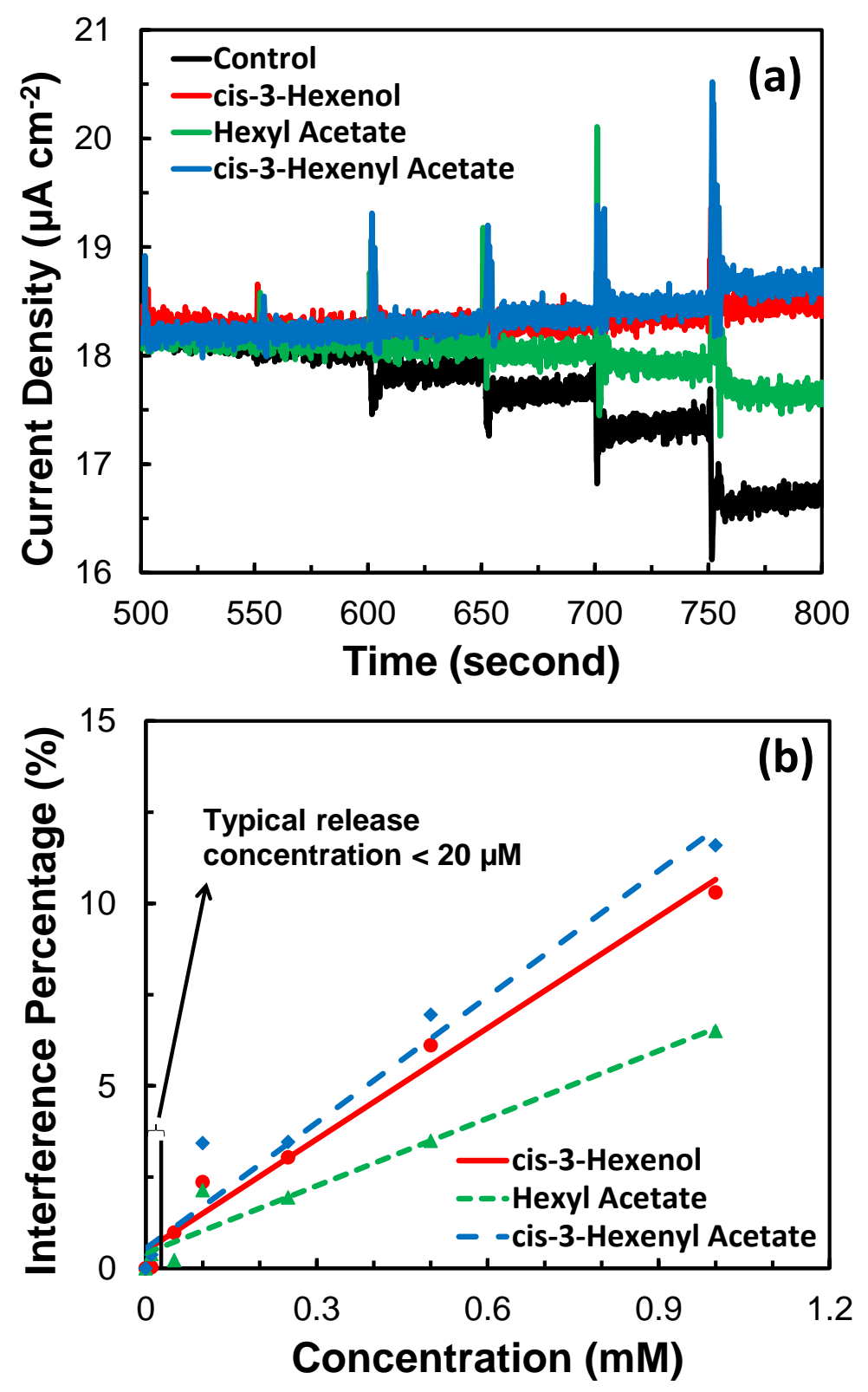

Figure 4: Interference of cis-3-hexenol, hexyl acetate and cis-3-hexenyl acetate on the amperometric measurements. Phosphate buffer is used as the control interfering compound (a). Percentage of interference caused by each compound at different concentrations (b). 


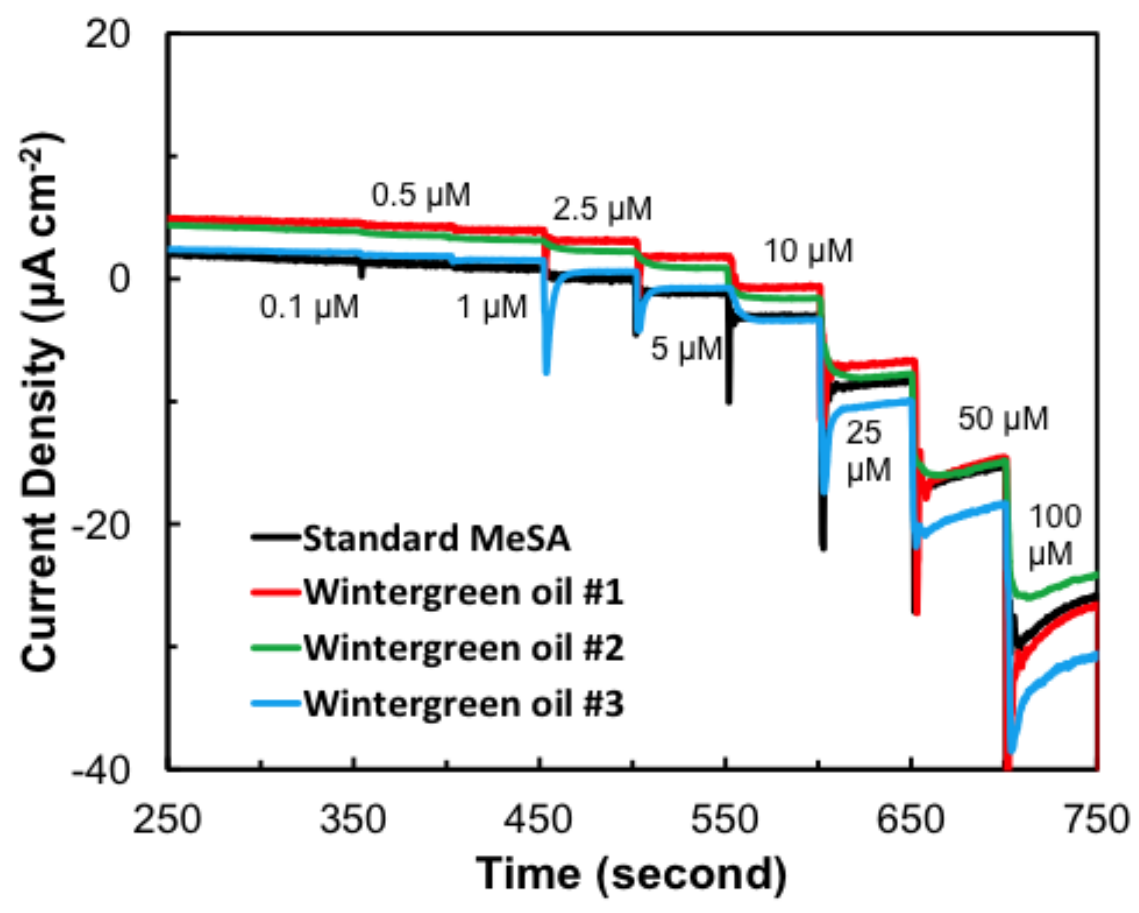

Figure 5: Constant potential amperometry of the electrode upon the stepwise additions of wintergreen oil ( 3 replicates) resulting in concentrations of $0.1,0.5,1,2.5,5,10,25,50,100 \mu \mathrm{M}$ (98\% MeSA) wintergreen oil. A control experiment using standard methyl salicylate at identical concentrations (black curve) is also shown for comparison. 


\section{Graphic for Table of Content}

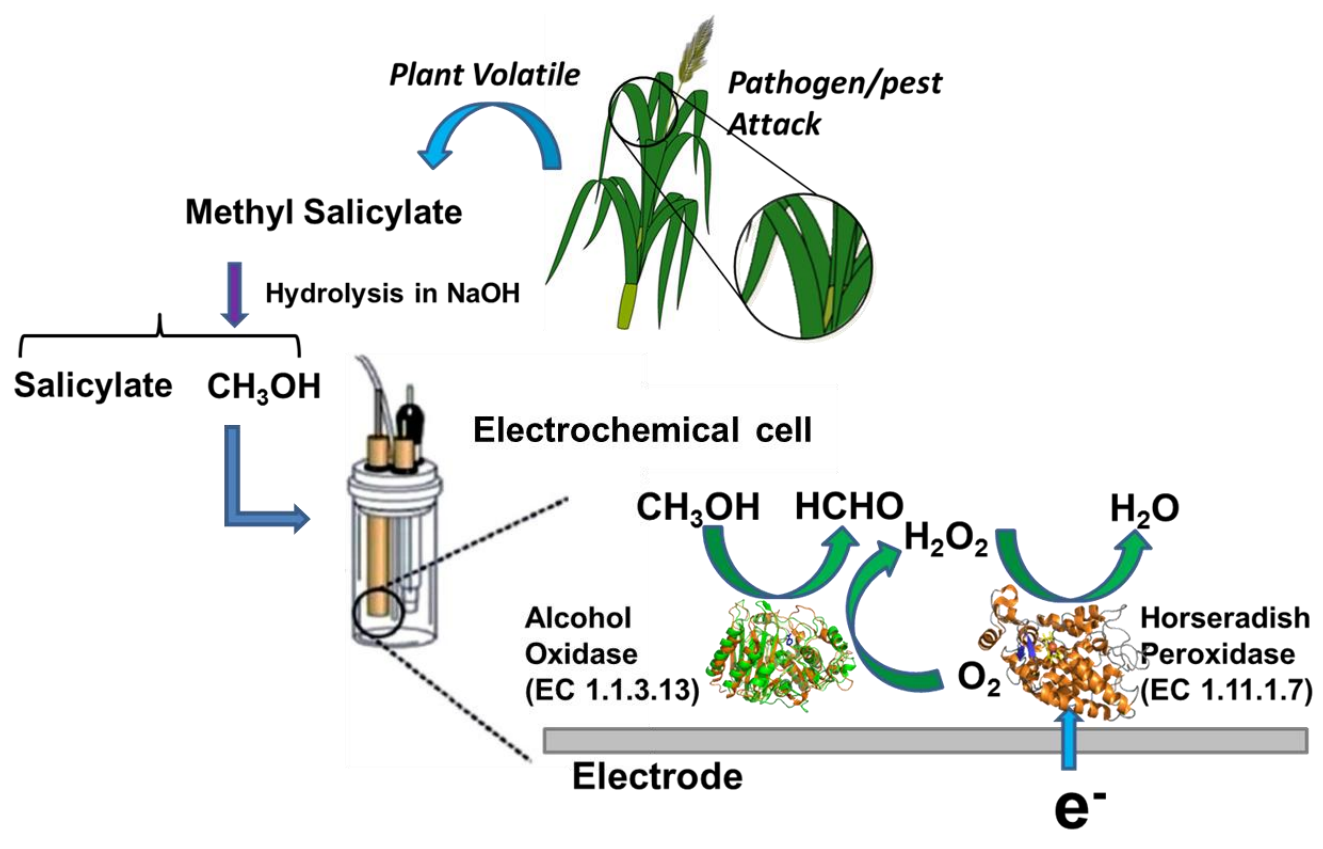

